\title{
The Application of Hybrid Interferometer-MUSIC to Eliminate Grating Lobe Effects
}

\author{
Robert Penno \\ University of Dayton \\ 300 College Park \\ Dayton, Ohio 45469-0226 \\ (937) 2293984 \\ Robert.Penno@notes.udavton.edu
}

\author{
Krish Pasala \\ University of Dayton \\ 300 College Park \\ Dayton, Ohio 45469-0226 \\ (937) 2292683 \\ Krish.Pasala@notes.udayton.edu
}

\author{
Stephen Schneider \\ United States Air Force \\ AFRL/SNRR \\ Wright-Patterson Air Force \\ WPAFB, Ohio \\ (937) 255-4120 \\ Stephen.Schneider@afrl.af.mil
}

\begin{abstract}
This paper first shows that grating lobes in a linear array can arise from the periodicity inherent in the steering vector of the Multiple Signal Classification (MUSIC) algorithm; that when that periodicity is destroyed, grating lobes are eliminated. This periodicity, and the resulting grating lobes, can be mitigated by using array elements with field patterns dissimilar in magnitude, phase or both. Simulations shown herein demonstrate this.

This principle then is applied to the Hybrid Interferometer by implementation of MUSIC (HybridMUSIC). Because of the differences in the modal field patterns of the multi-mode antenna (e.g. $\mathrm{N}$-arm spiral), the resulting steering vectors are sufficiently aperiodic as to reduce grating lobes to vestigial lobes. It is shown that Hybrid-MUSIC produces AOA estimates significantly improved over the linear phase interferometer (LPI) and the Hybrid Interferometer. Furthermore, this accuracy is maintained over a broad range of frequencies and SNR's, without evidence of the threshold effect present in both the LPI and the Hybrid Interferometer.. This method does require increased computation, but offers the potential to resolve multiple signals as well as mitigate interfering signals.
\end{abstract}

\section{TABLE OF CONTENTS}

1. INTRODUCTION.........................................1

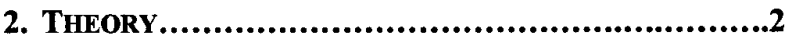

3. RESULTS..................................................5

4. Conclusions............................................ 7

5. REFERENCES.............................................7

6. BIOGRAPHIES........................................8

\section{INTRODUCTION $\mathbf{N}^{1,2,3}$}

The problem of identifying the Angle of Arrival (AOA) of harmonic electromagnetic waves from an unknown location has many applications in modern avionics. Typically, this

1 "U.S. Government work not protected by U.S. copyright."

2 IEEEAC paper \#1019, Updated July 24, 2002

${ }^{3}$ This work was supported by AFRL/SNRR, WPAFB, OH
AOA identification must be performed over a wide range of frequencies while using a minimal number of antennas. One of the most commonly employed systems used to determine AOA is the five element linear phase interferometer (LPI) [1]-[4], which consists of two distinct, three-element interferometers each of which determines a direction cosine relative to the baseline that defines that interferometer. From these direction cosines, the elevation and azimuth angles may be deduced trigonometrically. Focusing on the component, three-element interferometer, it is the largest element separation that simultaneously provides the excellent resolution of AOA, while also introducing the potential for gross error through ambiguous solutions. These ambiguous solutions arise from the grating lobe phenomena associated with any linear array where element spacings exceed one-half wavelength. The short baseline pair is used to resolve these ambiguities. However, the performance of the short baseline pair is very frequencydependent making the three-element interferometer an inherently narrow-band system. Consequently, to obtain accurate estimates of $\mathrm{AOA}$ over a wide band of frequencies, several different five-element interferometers are necessary to provide the required coverage over the whole band, thus necessitating a large "antenna farm" to be sited on the platform.

A more recent antenna technology, consisting of a single aperture, but supporting multiple arms such as spirals, has been developed [5]. This single aperture, multi-arm system, when excited properly, can support multiple modes. By comparing the modal outputs, it is possible to determine both the azimuth and the elevation angles of the incident radiation. In addition, the multi-mode antenna can be designed to operate over an arbitrarily wide bandwidth. Thus, this system makes it possible to obtain AOA estimates using a single aperture over a wide bandwidth. It has been demonstrated [6]-[7] that when this multi-arm antenna is coupled with the increasingly practical multi-channel receiver, the power and flexibility of modern signal processing methods can be brought to bear upon the angle estimation problem to obtain even better angle estimates. Nonetheless, the accuracy attainable with the multi-arm system is not comparable to that obtained using the conventional five-element interferometer. 
To take advantage of the broad frequency range over which the multi-mode antenna operates, while obtaining the accuracy provided by the long baseline of the interferometer, a Hybrid Interferometer was developed [8]. This hybrid replaces the short baseline pair, used for ambiguity resolution in the interferometer, with a single, wideband multi-mode antenna. Then, the coarser AOA estimate obtained from the multi-mode element is used to resolve the more accurate, but ambiguous AOA estimate obtained from the pair of multi-mode antennas which are separated by a long baseline. The ambiguities caused by the grating lobes which are presented by the long baseline still degrade AOA estimate performance, however. In fact, for a given long baseline, both the Hybrid Interferometer and the LPI exhibited a threshold Signal-to-Noise ratio (SNR) below which the ambiguity was unlikely to be accurately resolved, and an accurate estimate of AOA was not expected. Nonetheless, the Hybrid Interferometer was shown to produce AOA estimates with much lower variances and biases, over a significantly larger bandwidth, with a much lower threshold SNR than the LPI.

While the Hybrid Interferometer is a significant improvement over the LPI, the problem characterized by the threshold SNR reveals a systematic flaw in both systems that must be overcome to produce accurate and reliable AOA estimates. The source of this threshold problem, grating lobes, is a well understood phenomenon in the study of linear arrays [9]. However, in the application of parameter estimation techniques to single, multi-mode antennas [6]-[7], no such phenomenon was observed. If one considers the multi-mode antenna as an array of individual antennas, each characterized by a different modal field pattern, then it is quickly surmised that an array of equally spaced antennas, each with dissimilar field patterns, would likely exclude grating lobes, too. Moreover, when parameter estimation techniques, such as the MUSIC approach, are applied to a single multi-mode antenna, or a pair of multi-mode antennas separated by a long baseline, the periodicity of the resulting steering vector is eliminated. This effectively reduces grating lobes to lower level vestigial lobes. In the MUSIC pseudospectrum, such vestigial lobes are easily discerned from the actual main lobe associated with the AOA. In this work, the MUSIC algorithm is applied to the outputs of two multi-mode antennas, separated by a long baseline. The results, when viewed over a range of SNR's, frequency, and long baselines, reveal that grating lobes, and the associated threshold SNR phenomenon, are eliminated, with a resulting improvement in AOA estimates over both Hybrid Interferometer and the LPI. Statistics of the angle estimates for various cases are presented. Extended applications, such as multiple signals or mitigation of interference, are discussed.

\section{THEORY:}

Before discussing the application of MUSIC to the Hybrid Interferometer (Hybrid-MUSIC), it is useful to consider the linear antenna array and the growth of grating lobes when element spacing exceeds one-half wavelength. It is helpful to understand the grating lobe phenomenon (in the context of the periodicity of linear arrays) as a prelude to the application of MUSIC to the Hybrid Interferometer.

\section{Grating Lobes in a Linear Array Using MUSIC}

Consider an array of single mode antennas as shown in Figure 1, where the field pattern of the $\mathrm{m}^{\text {th }}$ antenna is given by $f_{m}(\theta, \phi)$. If $\vec{g}$ is the steering vector associated with the outputs of the antennas comprising the array, and $\overrightarrow{\boldsymbol{\eta}}$ is the noise vector associated with terminal outputs of these antennas, then the output signal vector, $\overline{\mathbf{x}}$, is given by

$$
\overrightarrow{\mathbf{x}}=\overrightarrow{\mathbf{g}}+\overrightarrow{\boldsymbol{\eta}}
$$

where the $\mathbf{m}^{\text {th }}$ element of $\overline{\mathbf{g}}$ is given by

$$
\begin{gathered}
g_{m}\left(\theta_{s}, \varphi_{s}\right)=f_{m}\left(\theta_{s}, \varphi_{s}\right) E_{o} e^{j(m-1) \beta d \sin \left(\theta_{s}\right) \sin \left(\varphi_{s}\right)} \\
=f_{m}\left(\theta_{s}, \varphi_{s}\right) E_{o} e^{j \psi_{m}\left(\theta_{s}, \varphi_{s}\right)}
\end{gathered}
$$



Figure 1: Linear Array

where $E_{0}$ is the magnitude of the incident wave. It is assumed that the noise present is white, Gaussian and zeromean. Then, the autocorrelation matrix is given as

$$
\mathbf{R}_{\overline{\mathbf{x}}}=\mathbf{E}\left\{\overrightarrow{\mathbf{x}}^{\mathbf{H}}\right\}
$$

which is an (M x M) matrix. The noise vectors, collected together in the noise matrix, $Q_{N}$, can be found accordingly. Using equation 2 to describe the elements of the steering vector, evaluated at $\left(\theta_{\mathrm{s}}, \phi_{\mathrm{s}}\right)$, the MUSIC spectrum is seen to be

$$
P_{\text {MUSIC }}=\frac{\left(\overline{\mathbf{g}}^{\mathbf{H}} \overrightarrow{\mathbf{g}}\right)}{\left(\overrightarrow{\mathbf{g}}^{\mathbf{H}} \mathbf{Q}_{\mathrm{N}}\right)\left(\mathbf{Q}_{\mathbf{N}}^{\mathbf{H}} \overrightarrow{\mathrm{g}}\right)}
$$


Equation 4 can be rearranged and written as

$$
P_{\text {MUSIC }}=\frac{\left(\vec{g}\left(\theta_{s}, \varphi_{s}\right)^{H} \vec{g}\left(\theta_{s}, \varphi_{s}\right)\right)}{\vec{g}\left(\theta_{s}, \varphi_{s}\right)^{H}\left(Q_{N}(\theta, \varphi) Q_{N}^{H}(\theta, \varphi)\right) \vec{g}\left(\theta_{s}, \varphi_{s}\right)}
$$

so that the denominator of equation 5 can be written as

$$
\begin{aligned}
& \vec{g}\left(\theta_{s}, \varphi_{s}\right)^{H} N_{m, l} \bar{g}\left(\theta_{s}, \varphi_{s}\right) \\
= & \sum_{m=1}^{M} g_{m}^{*}\left(\theta_{s}, \varphi_{s}\right) \sum_{l=1}^{M} N_{m, 1} g_{l}\left(\theta_{s}, \varphi_{s}\right) \\
= & \sum_{m=1}^{M} f_{m}^{*}\left(\theta_{s}, \varphi_{s}\right) e^{-j \psi(m)} \sum_{l=1}^{M} N_{m, 1} f_{l}\left(\theta_{s}, \varphi_{s}\right) e^{j \psi(l)}
\end{aligned}
$$

where

$$
\begin{aligned}
& \psi(\mathbf{m})=(\mathbf{m}-\mathbf{1}) \beta d \sin \left(\theta_{s}\right) \sin \left(\varphi_{s}\right) \\
& \mathbf{N}_{m, 1}=\left\{\mathbf{Q}_{N}(\theta, \varphi) \mathbf{Q}_{N}^{H}(\theta, \varphi)\right\}_{m, I}
\end{aligned}
$$

In the event that $f_{m}\left(\theta_{s}, \phi_{s}\right)$ is identical for all of the antennas, the term, $\left|f_{m}\left(\theta_{s}, \phi_{s}\right)\right|^{2}$ factors out of both numerator and denominator, cancels, and eliminates the dependence of the pseudospectrum upon the element field pattern. Periodicity appears when the remaining portion of the steering vector, $\overrightarrow{\mathbf{g}}$, is periodic, i.e.

$$
\begin{gathered}
\beta d \sin \left(\theta_{\mathrm{s} 1}\right) \sin \left(\varphi_{\mathrm{s} 1}\right)=\beta d \sin \left(\theta_{\mathrm{s} 2}\right) \sin \left(\varphi_{\mathrm{s} 2}\right)+2 \mathrm{p} \pi \\
\mathrm{p}=\ldots-2,-1,0,1,2,3, \ldots
\end{gathered}
$$

If, on the other hand, $f_{m}\left(\theta_{s}, \phi_{s}\right)$ is not identical in magnitude and phase, the periodicity is eliminated by the non-uniform weighting affected by the field patterns in the denominator. To better describe this phenomenon, consider a five-element linear array, comprised of $\mathrm{z}$-directed dipole antennas, oriented along the $y$-axis as in Figure 1, with field pattern characterized by

$$
f_{m}(\theta, \varphi)=\sin (\theta)
$$

If the spacing of these antennas is $1 \lambda$, a single grating lobe is expected. The MUSIC pseudospectrum of Figure 2 shows the presence of a grating lobe for the case of $\theta=$ $40^{\circ}$ and $\mathrm{SNR}=10 \mathrm{~dB}$, with a grating lobe at $\theta_{\mathrm{g}}=-20.92^{\circ}$.



Figure 2: Five-Element Linear Array with Sinusoidal Field Pattern.
When the elements of the array are replaced by antennas, where the $\mathrm{m}^{\text {th }}$ element of the array has a field pattern described by

$$
f_{m}(\theta, \varphi)=\cos (\theta) \cdot\left(J_{m+1}(m \sin (\theta))+J_{m-1}(m \sin (\theta))\right),
$$

Here, $\mathrm{J}_{\mathrm{m}}(\theta)$ is the $\mathrm{m}^{\text {th }}$ order Bessel function of the first kind, the next largest side lobe (not a grating lobe) is at least 15 $\mathrm{dB}$ below the main lobe as shown in the MUSIC pseudospectrum, Figure 3.

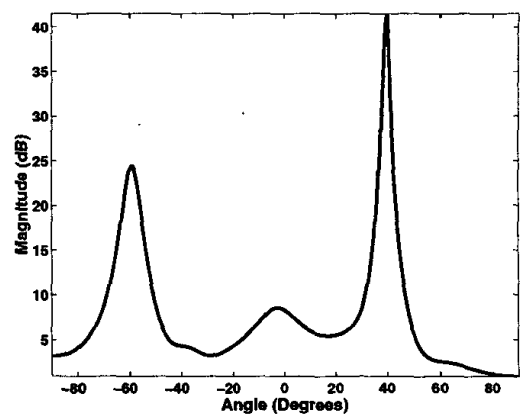

Figure 3: Five-Element Linear Array with Modal Bessel Field Pattern.

While this antenna field pattern was chosen because it emulates the modal field patterns of the multi-mode spiral antenna, used in the analysis of the Hybrid MUSIC interferometer, it is important to note that it establishes a unique field pattern at the location of each element of the array. Similar observations are found when using dissimilar phase, e.g.

$$
f_{m}(\theta, \varphi)=e^{j m \theta}
$$

As described in [7], each mode of the multi-mode antenna can be construed as an individual antenna, each of which has a different field pattern. Each multi-mode antenna (e.g. an N-arm spiral antenna), then, acts as a collection of (N-1) different antennas.

\section{Application of MUSIC to the Hybrid Interferometer}

In the case of the five-element interferometer (Figure 4), element- 1 in combination with element- 2 and element- 4 forms a pair of short baseline interferometers. These short baseline interferometers help to resolve the phase ambiguities associated with the long baseline interferometers formed by elements- 1 and 3 and elements- 1 and 5. For high probability of ambiguity resolution, it is desirable that the inter-element spacing in the short baseline interferometers be a half wavelength or less. This requirement makes it difficult to design a five-element interferometer that operates over a wide bandwidth. 
Figure 4 shows a typical five-element interferometer, where element-1 combines with element- 2 to form a short baseline. This coarse estimate of $\gamma$ is used to resolve the multiple estimates of $\gamma$ obtained from the long baseline pair, comprised of element-1 and element-3. Likewise, element1 and element- 4 form a short baseline and provide a coarse estate of angle, $\alpha$, that resolves the multiple estimates of $\alpha$ obtained from the long baseline pair of element-1 and element-5. To achieve the highest probability of ambiguity resolution from this system, the short baseline spacing must be one-half wavelength or less. In many situations, this is impractical, if not impossible, especially over a wide bandwidth.

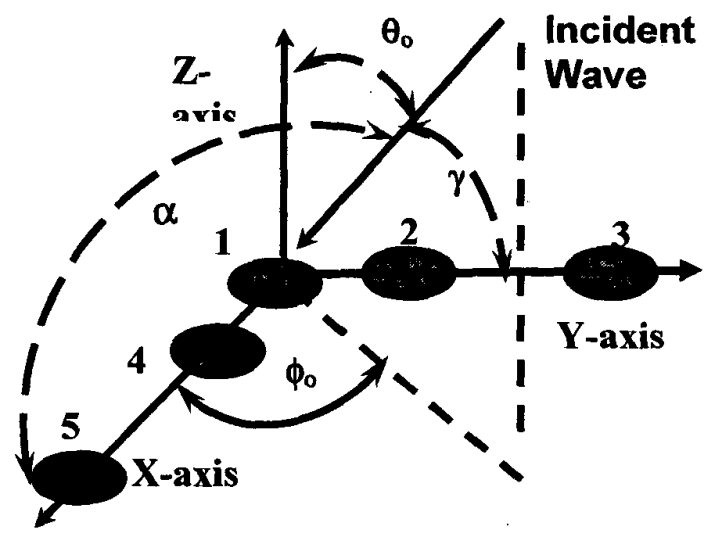

Figure 4: Five-Element, Conventional Interferometer

As described in [8], the three element hybrid consists of three identical multi-mode antennas, arranged along orthogonal baselines as shown in Figure-5. Estimates of $\gamma$ are obtained from the modal outputs of both elements 1 and 3 , while estimates of $\alpha$ are obtained from the modal outputs of both elements 1 and 2 .

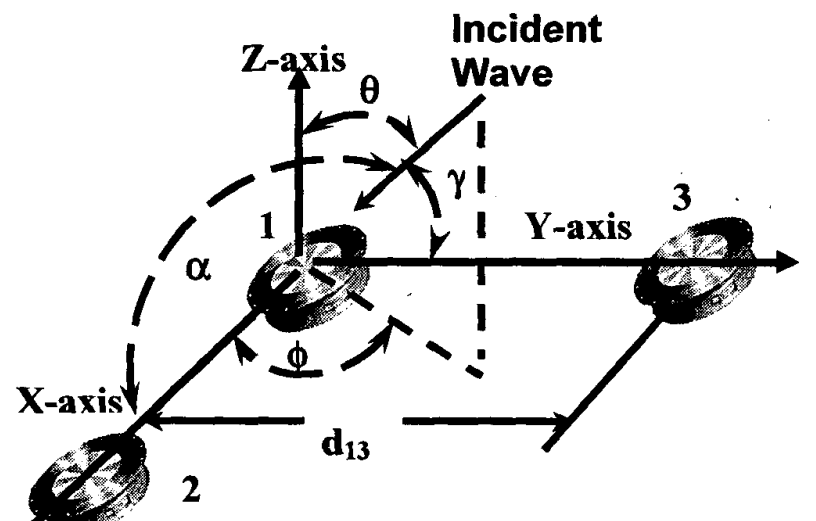

Figure 5: Three-Element Hybrid Interferometer

As in [7], the MUSIC approach is implemented in two steps. First, the data space is decomposed into two subspaces: a signal-plus-noise subspace and a noise subspace. Secondly, it may be recognized that the steering vector corresponding to the true AOA is orthogonal to this noise subspace. This leads to the definition of a pseudospectrum, the peak of which corresponds to an estimate of the AOA. The MUSIC approach may be developed using either terminal or modal output voltages; the formulation developed here uses the modal output voltages directly. As described in [7], they are related by a linear transformation.

\section{The Signal Model}

Analysis will be focused upon a single baseline system, located along the y-axis. This system is comprised of element- 1 and element-3 of Figure 5. For such a system, assume that there are $\mathrm{K}$ sources and consider the signal due to the $\mathbf{k}^{\text {th }}$ source, which is either $\theta$ - or $\phi$-polarized. The modal output vector, then, is given by:

$$
\overrightarrow{\mathbf{M}}_{k}=\mathbf{a}_{\mathbf{k}} \overrightarrow{\mathrm{g}}_{\mathrm{k}}\left(\theta_{\mathrm{k}}, \varphi_{\mathrm{k}}\right)
$$

where the angular dependence of the $n^{\text {th }}$ element of the vector, $\overrightarrow{\mathbf{g}}_{\mathbf{k}}$, is given by:

$$
\begin{aligned}
& \mathbf{g}_{\theta_{k}}^{n}=e^{-j n \varphi_{k}}\left[\cos \theta_{k}\left(J_{n-1}\left(n \sin \theta_{k}\right)+J_{n+1}\left(n \sin \theta_{k}\right)\right)\right] \\
& g_{\varphi_{k}}^{n}=e^{-j n \varphi_{k}}\left[\left(J_{n+1}\left(n \sin \theta_{k}\right)-J_{n-1}\left(n \sin \theta_{k}\right)\right)\right]
\end{aligned}
$$

for the $\theta$ - and the $\phi$-polarizations, respectively, and

$$
\mathbf{a}_{\mathrm{k}}=\mathbf{E}_{\mathbf{0}, \mathrm{k}} \mathbf{e}^{j \psi_{0, \mathrm{k}}}
$$

is the complex phase associated with each mode.

While equation 13 describes the modal output voltages of the multi-mode- 1 , located at the origin, those at multi-mode -2 , located on the $y$-axis at $y=d_{13}$, are given by

$$
\overrightarrow{\mathbf{M}}_{k, 2}=\overrightarrow{\mathbf{M}}_{k, 1} \mathrm{e}^{\mathrm{j} \beta d \sin \left(\theta_{k}\right) \sin \left(\varphi_{k}\right)}
$$

The complete, concatenated modal output voltage, $\overrightarrow{\mathbf{M}}_{\mathbf{k}}$ due to the $k^{\text {th }}$ signal is given by the $(2 \mathrm{~N} \mathrm{x} 1)$ column vector

$$
\overrightarrow{\mathbf{M}}_{\mathbf{k}}=\left[\overrightarrow{\mathbf{M}}_{\mathbf{k}, \mathbf{1}}, \overrightarrow{\mathbf{M}}_{\mathbf{k}, \mathbf{2}}\right] \text {. }
$$

The total modal output voltage vector due to all of the sources is given by

$$
\overrightarrow{\mathbf{M}}_{\mathbf{s}}=\sum_{\mathbf{k}=1}^{\mathbf{K}} \mathbf{M}_{\mathbf{k}}=\sum_{\mathbf{k}=1}^{\mathrm{K}} \mathbf{a}_{\mathbf{k}} \overrightarrow{\mathbf{g}}_{\mathbf{k}}
$$

Adding zero-mean, white, Gaussian noise of variance, $\boldsymbol{\sigma}_{\eta}^{2}$, to the modal output(s) produces the total output vector

$$
\overrightarrow{\mathbf{M}}_{\mathbf{T}}=\overrightarrow{\mathbf{M}}_{\mathbf{s}}+\overrightarrow{\boldsymbol{\eta}}
$$


The Correlation Matrix

The correlation matrix, $\mathbf{R}_{\overline{\mathbf{M}}_{\mathbf{T}}}$, is defined as

$$
\mathbf{R}_{\overrightarrow{\mathbf{M}}_{\mathbf{T}}}=\mathbf{E}\left|\overrightarrow{\mathbf{M}}_{\mathbf{T}} \overrightarrow{\mathbf{M}}_{\mathbf{T}}^{\mathbf{H}}\right|
$$

Assuming that the noise is uncorrelated with the signal, and that noise in each channel is independent, equation 20 reduces to

$$
\mathbf{R}_{\overline{\mathbf{M}}_{\mathrm{T}}}=\mathbf{R}_{\overline{\mathbf{M}}_{\mathrm{s}}}+\boldsymbol{\sigma}_{\eta}^{2} \mathbf{I}
$$

The true signal correlation matrix, $\mathbf{R}_{\overrightarrow{\mathbf{M}}_{\mathrm{s}}}$, is given by

$$
\mathbf{R}_{\overrightarrow{\mathrm{V}}_{\mathrm{s}}}=\mathrm{E}\left[\overrightarrow{\mathrm{V}}_{\mathrm{s}} \overrightarrow{\mathrm{V}}_{\mathrm{s}}^{\mathrm{H}}\right]=\mathrm{E}\left[\left(\sum_{\mathrm{k}=1}^{\mathrm{K}} \mathbf{a}_{\mathrm{k}} \overrightarrow{\mathrm{g}}_{\mathrm{k}}\right)\left(\sum_{\mathrm{k}=1}^{\mathrm{K}} \mathrm{a}_{\mathrm{k}} \overrightarrow{\mathrm{g}}_{\mathrm{k}}\right)^{\mathrm{H}}\right]
$$

If the external sources are uncorrelated, i.e.

$$
\mathbf{E}\left[\mathbf{a}_{\mathbf{k}} \mathbf{a}_{\mathbf{l}}\right]=\mathbf{0}, \text { for } k \neq \mathbf{l},
$$

then, some straight forward manipulation produces

$$
\begin{aligned}
& \mathbf{R}_{\overrightarrow{\mathrm{V}}_{\mathrm{s}}}=\mathbf{R}_{\mathrm{s}} \mathbf{P R}_{\mathrm{s}}^{\mathrm{H}}, \\
& \mathbf{R}_{\mathrm{S}}=\left[\begin{array}{ccc}
\vdots & \vdots & \vdots \\
\overrightarrow{\mathbf{g}}_{1}, \overrightarrow{\mathbf{g}}_{2} & \cdots \overrightarrow{\mathbf{g}}_{\mathrm{K}} \\
\vdots & \vdots & \vdots
\end{array}\right] \\
& \text { and } \mathbf{P}=\operatorname{diag}\left[\begin{array}{llll}
\mathbf{P}_{1} & \mathbf{P}_{2} & \cdots & \mathbf{P}_{\mathrm{K}}
\end{array}\right]
\end{aligned}
$$

Note that $R_{\mathrm{s}}$ is a ( $\left.2 \mathrm{~N} \times \mathrm{K}\right)$ matrix. Also, note that the rank $\left(R_{s}\right)$ is $K$, and is assumed to be less than $2 N$. Rather, the number of sources must be less than the total number of modal outputs available from the two multi-mode antennas. The elements of $P$ are the average powers of the $K$ external sources and are given by:

$$
\mathbf{P}_{\mathbf{k}}=\mathbf{E}\left(\left|\mathbf{a}_{\mathbf{k}}\right|^{2}\right)
$$

This expression for the correlation matrix, equation 24 , has exactly the same form as the correlation matrix of a linear array of $2 \mathrm{~N}$ elements [10], [11] illuminated by $\mathrm{K}$ uncorrelated sources. Thus, it follows [11] that an eigendecomposition of the correlation matrix reveals the presence of the K-signal eigenvalues and $(2 \mathrm{~N}-\mathrm{K})$ noise eigenvalues equal to $\sigma_{\eta}^{2}$. In addition, it may be shown that the steering vector corresponding to any of the true signals is orthogonal to the subspace spanned by the noise eigenvectors. This observation leads to the formulation of the MUSIC pseudospectrum.

\section{MUSIC Pseudospectrum}

The eigen-decomposition of the estimated correlated matrix is

$$
\hat{\mathrm{R}}=\mathrm{Q} \Lambda \mathrm{Q}^{\mathrm{H}}
$$

The eigenvalues contained in $\Lambda$ allow for the separation and identification of the noise and signal+noise subspaces. The matrix $Q$ consists of the $K$ "signal" eigenvectors and $(2 \mathrm{~N}-\mathrm{K})$ noise eigenvectors. Let the $(2 \mathrm{~N}-\mathrm{K})$ noise eigenvectors be grouped together in a noise matrix defined by

$$
\mathbf{Q}_{N}=\left|\overrightarrow{\mathbf{q}}_{K+1}, \overrightarrow{\mathbf{q}}_{K+2}, \ldots, \overrightarrow{\mathbf{q}}_{2 N}\right| \text {. }
$$

Then, the MUSIC spectrum is given by

$$
\begin{aligned}
\mathbf{P}_{\text {Music }}(\theta, \varphi) & =\frac{1}{\sum_{1=\mathrm{K}+1}^{M} \mid \overrightarrow{\mathbf{q}}_{\mathbf{l}}^{\mathbf{H}}\left(\left.\hat{\mathbf{g}}(\theta, \varphi)\right|^{2}\right.} \\
& =\frac{1}{\hat{\mathbf{g}}^{\mathbf{H}}(\theta,)\left(\mathbf{Q}_{\mathrm{N}} \mathbf{Q}_{\mathrm{N}}^{\mathbf{H}}\right) \hat{\mathbf{g}}(\theta,)}
\end{aligned}
$$

where $\hat{\mathbf{g}}$ is the normalized steering vector given by

$$
\hat{\mathbf{g}}=\frac{\overrightarrow{\mathbf{g}}}{\|\overrightarrow{\mathbf{g}}\|}
$$

Here $\overline{\mathbf{g}}(\boldsymbol{\theta}, \varphi)$ is the steering vector corresponding to any possible AOA, $(\theta, \phi)$, while $\|\overrightarrow{\mathbf{g}}\|$ is the norm of this steering vector. When this angle coincides with the true angle of arrival of any of the $K$ signals, the denominator tends to a small value resulting in a large peak in the pseudospectrum. It is important to note that computationally it is advantageous to determine the noise subspace from a singular value decomposition of the data matrix rather than the estimated correlation matrix.

\section{RESULTS}

Shown below is an elevation pseodospectrum obtained using the Hybrid MUSIC approach for the case $\phi=90^{\circ}$, $\mathrm{SNR}=10 \mathrm{~dB}$ and $\mathrm{d}_{\max }=1 \lambda$ which is comparable to the example employed using the linear array displayed in Figure 2 and 3 . No grating lobes are present, having been extinguished by the dissimilar modal field patterns. More explicit suppression of the grating lobes is seen in the case of $d_{\max }=18.2 \lambda$ shown in Figure 7 . Here, the pseodospectrum contains eighteen lobes, one of which, located at the angle of incidence, is evidently dominant. 
The remaining vestigial lobes are at least $13 \mathrm{~dB}$ below the peak lobe.

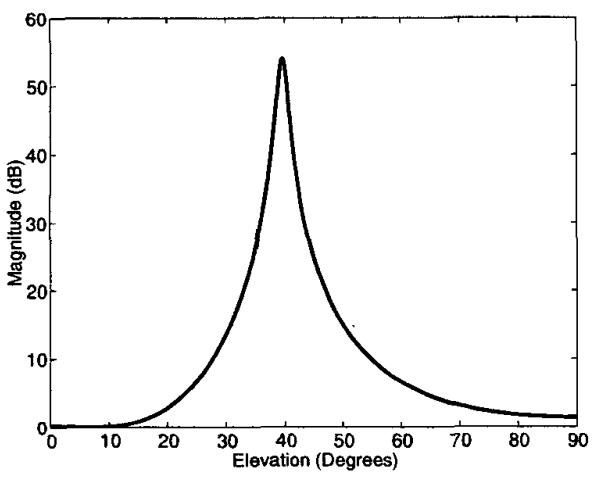

Figure 6: Three Element Hybrid $\left(\phi=90^{\circ}, 10 \mathrm{~dB}, d_{\max }=1 \lambda\right)$

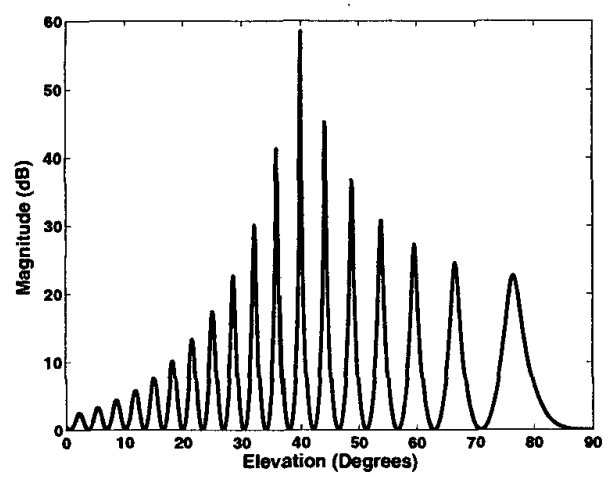

Figure 7: Three Element Hybrid $\left(\phi=90^{\circ}, 10 \mathrm{~dB}, d_{\max }=18.2 \lambda\right)$

\section{Effects of Variation with SNR}

In [8], it was observed that the classical interferometer, as well as the conventional hybrid, manifests a threshold SNR which is a function of long baseline and the width of the distribution of AOA estimates obtained from the small baseline pair or the multi-mode antenna. Both of these systems experience catastrophic failure when the distribution used to resolve ambiguity is wide enough to include several ambiguous solutions provided by the long baseline pair. As described earlier, Hybrid-MUSIC does not experience this catastrophic failure. Shown in Figure 8 are the variations of $\mathrm{AOA}$ standard deviation and bias as a function of SNR for the case of $\theta=40^{\circ}, \phi=90 .^{\circ}$ and $d_{\max }=$ 18.2 $\lambda$. In the case of both the LPI and the conventional Hybrid, this threshold is observed at approximately $17 \mathrm{~dB}$ and $14 \mathrm{~dB}$, respectively, while Hybrid MUSIC shows excellent statistics over the entire range of SNR with no threshold effects apparent.



Figure 8a: Error Standard Deviation vs. $\operatorname{SNR}\left(d_{\max }=18.2 \lambda\right)$

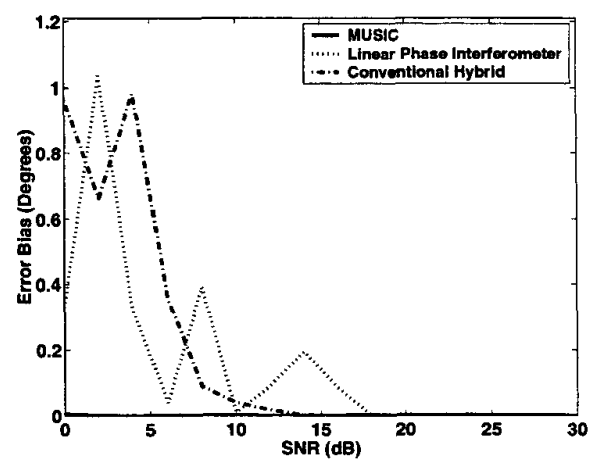

Figure 8b: Error Bias vs. SNR

$$
\left(d_{\max }=18.2 \lambda\right)
$$

Effects of Variation with Frequency

As mentioned previously, applications exist where AOA information is required over a large range of frequencies. When using the LPI, a number of 5-element configurations are required to achieve the desired accuracy. Using the Hybrid, however, takes advantage of the inherent frequency independence of the multi-mode antennas, requiring three wide-band multi-mode antennas to complete the task. In Figure 9, variation of standard deviation and bias with respect to frequency is shown for the LPI, conventional hybrid and the Hybrid-MUSIC for the case of $\theta=40^{\circ}, \phi=$ $90 .^{\circ}, \mathrm{d}_{\max }=18.2 \lambda$ and $\mathrm{SNR}=0 \mathrm{~dB}$ for the range of $2 \mathrm{GHz}$ to $18 \mathrm{GHz}$. It is seen that the conventional hybrid performs consistently over the frequency band, while the LPI sees serious performance degradation at low frequency. 


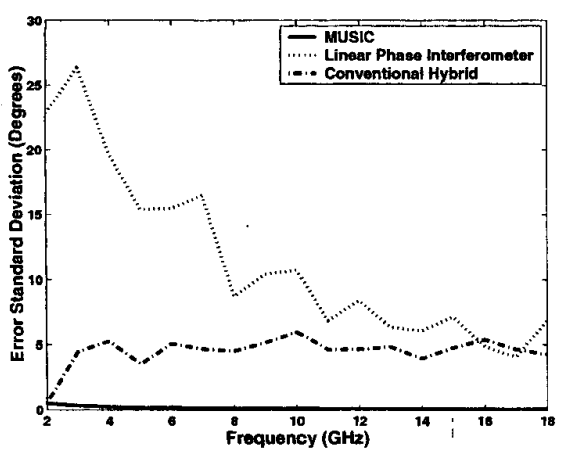

Figure 9a: Error Standard Deviation vs. Frequency $\left(d_{\max }=18.2 \lambda, S N R=0 \mathrm{~dB}\right)$

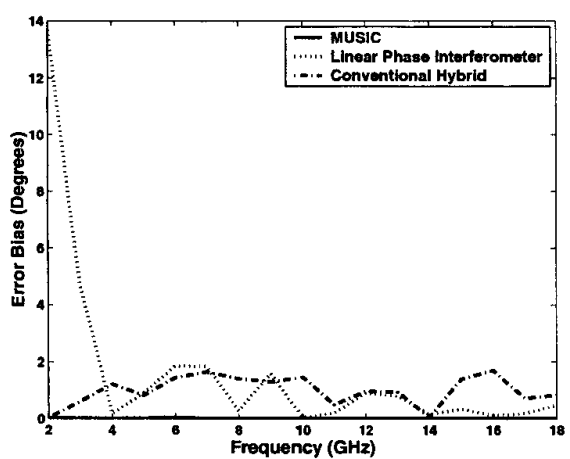

Figure 9b: Error Bias vs. Frequency $\left(d_{\max }=18.2 \lambda, S N R=0 \mathrm{~dB}\right)$

The Hybrid-MUSIC, on the other hand, consistently provides superior AOA estimates. Moreover, HybridMUSIC outperforms both the LPI and the conventional hybrid over a wide frequency bands.

\section{CONCLUSIONS}

This work shows the application of the parameter estimation technique, MUSIC, to the hybrid multi-mode interferometer for the determination of AOA. The primary source of error in any interferometer is the resolution of ambiguous solutions, referred to otherwise as grating lobes. It is seen that grating lobes arise naturally when MUSIC is applied to a linear array (with spacing in excess of one-half wavelength) through the periodicity of the steering vector used to describe the MUSIC pseudospectrum. Furthermore, these grating lobes are eliminated using elements with dissimilar field patterns (phase, magnitude or both).

The conventional hybrid multi-mode interferometer is an improvement over the conventional LPI in that it provides improved accuracy over a wide range of frequencies, while using significantly fewer apertures. Since the hybrid multi- mode interferometer utilizes two multi-mode antennas spaced more than one-half wavelength apart, however, grating lobes are present and limit its ability to produce accurate determination of AOA. Application of MUSIC to the modal outputs (or, equivalently, to the terminal outputs) takes advantage of the dissimilar modal field patterns to eliminate the periodicity in the steering vector. Thus, grating lobes in the MUSIC pseudospectrum are reduced to vestigial lobes.

Also eliminated is the threshold effect evident in both the LPI and the conventional hybrid. As described in [8], and evident in Figure 8, the threshold effect results from inaccurate ambiguity resolution. The probability density function (p.d.f.) associated with the main lobe and associated grating lobes are very closely spaced for long baselines, while the p.d.f. associated with the AOA derived from either a small baseline interferometer or a single multimode antenna widens with increased noise (lower SNR). The gross error associated with AOA estimates made below the SNR threshold, then result the selection of a grating lobe to produce the AOA estimate instead of the main lobe. Since the periodicity that produces these grating lobes has been eliminated, no such ambiguity resolution is required.

Moreover, excellent results are obtained by Hybrid-MUSIC over a wide range of SNR's and frequencies, while retaining all of the advantages of the conventional Hybrid. AOA determination is performed over these parameters without regard to resolution of unwanted ambiguities. In [12] it was shown that it is possible to obtain good estimates from a single, multi-mode antenna in the presence of jamming. It is possible to apply these same principles to obtain even better angle estimates from a Hybrid-MUSIC system in the presence of jamming. Finally, the ability to discern multiple signals is inherent to MUSIC, and has been included in the signal model described herein.

\section{REFERENCES:}

[1] Jacobs, E.A., and Ralston, E.W., "Ambiguity resolution in Interferometry," IEEE Transactions on Aerospace and Electronic systems, Vol. AES-17, No.6, pp.766780, Nov. 1981.

[2] Hansen, R.C (Ed). Microwave Scanning Antennas, Vol. III: Array Systems, New York: Academic Press, 1966.

[3] Stansfield, R.G., "Statistical Theory of D.F. fixing," Journal of IEE, (London), 94 (k) 3A, 762-770.

[4] Kendall,W.B., "Unambiguous accuracy of an interferometer angle-measuring system," IEEE Transactions on Space Electronics and Telemetry, SET11, pp.62-70, June 1965.

[5] Corzine, R.G, and Mosko J.A., "Four-Arm Spiral Antennas", Artech House, 1990, Norwood, Ma.

[6] Penno, R. P, and Pasala, K.M., "A Theory of Angle Estimation using a Multi-Arm Spiral Antenna," 
[7] Proceedings of IEEE Aerospace Conference held at Snowmass, Colorado, Mar. 6-13, 1999.

[8] Penno, R. P, and Pasala, K.M., "A Theory of Angle Estimation using a Multi-Arm Spiral Antenna," IEEE Transactions on Aerospace and Electronics Systems, Vol. 37, no. 1 .

[9] Penno, R. P, Pasala, K.M, and Stephen Schneider, "A Novel Multi-Mode Interferometer System," Proceedings of IEEE Aerospace Conference, Big Sky, Montana, March, 2001.

[10]W. Stutzman, G. Thiele, "Antenna Theory and Design,", J. Wiley \& Sons, New York, $2^{\text {nd }}$ Edition, 1998

[11] Johnson, D.H., Dudgeon, D.E.(1993), Array Signal Processing, Englewood Cliffs, NJ: Prentice Hall, 1993.

[12] Schmidt, R.O.(1981), A Signal Subspace Approach to Multiple Emitter Location and Spectral Estimation, $\mathrm{Ph} . \mathrm{D}$. dissertation, Stanford University, Stanford, CA, 1981

[13]Penno, R. P, Pasala, K.M. and Stephen Schneider, "Mitigation of Jamming of an Angle Estimation System Using Multi-mode Antennas," Proceedings of IEEE Aerospace Conference held at Big Sky, Montana, March, 2002

\section{BIOGRAPHIES:}



Dr. Krishna M. Pasala is a Professor in the Department of Electrical and Computer Engineering at the University of Dayton, Dayton, Ohio. He received the B.E. degree from Andhra University in 1970 and the Ph.D. in Electronics and Communications from the Indian Institute of Science in 1975. Dr. Pasala is a senior member of IEEE.

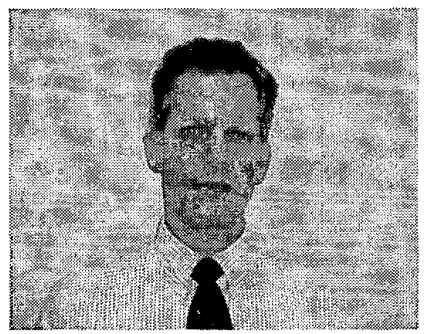

Dr. Robert Penno is an Associate Professor in the Department of Electrical and Computer Engineering at the University of Dayton, Dayton, Ohio. He received a
B.S.M.E. degree from Rose Polytechnic Institute in 1971, the M.S.E.E. from Rose-Hulman Institute of Technology in 1984, and the Ph.D. from the University of Dayton in 1987. $H e$ is a member of Sigma Xi and a senior member of IEEE.

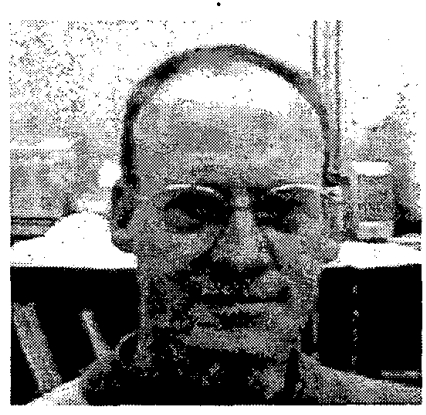

Dr. Stephen Schneider is the team leader for the Applied $R F$ Aperture Technology group in the Sensors directorate of the Air Force Research Laboratory, located at Wright Patterson Air Force Base, Dayton, Ohio. He received the B.S.E.E. from Arizona State University, and the M.S.E.E and Ph.D. in Electrical Engineering from the Ohio State University. He is a senior member of IEEE. 